\title{
Synthesis of thiolated, PEGylated and POZylated silica nanoparticles and evaluation of their retention on rat intestinal mucosa in vitro
}

Article

Accepted Version

Creative Commons: Attribution-Noncommercial-No Derivative Works 4.0

Ways, T. M. M., Lau, W. M., Ng, K. W. and Khutoryanskiy, V. V. (2018) Synthesis of thiolated, PEGylated and POZylated silica nanoparticles and evaluation of their retention on rat intestinal mucosa in vitro. European Journal of Pharmaceutical Sciences, 122. pp. 230-238. ISSN 0928-0987 doi: https://doi.org/10.1016/j.ejps.2018.06.032 Available at https://centaur.reading.ac.uk/78444/

It is advisable to refer to the publisher's version if you intend to cite from the work. See Guidance on citing.

To link to this article DOI: http://dx.doi.org/10.1016/j.ejps.2018.06.032

Publisher: Elsevier

All outputs in CentAUR are protected by Intellectual Property Rights law, including copyright law. Copyright and IPR is retained by the creators or other copyright holders. Terms and conditions for use of this material are defined in the End User Agreement. 


\section{www.reading.ac.uk/centaur}

\section{CentAUR}

Central Archive at the University of Reading

Reading's research outputs online 
Synthesis of thiolated, PEGylated and POZylated silica nanoparticles and evaluation of their retention on rat intestinal mucosa in vitro

Twana Mohammed M. Ways ${ }^{\mathrm{a}}$, Wing Man Lau ${ }^{\mathrm{b}}$, Keng Wooi $\mathrm{Ng}^{\mathrm{b}}$ and Vitaliy V.

Khutoryanskiy ${ }^{a *}$

${ }^{a}$ Reading School of Pharmacy, University of Reading, Whiteknights, Reading, RG6 6AD, United Kingdom

${ }^{\mathrm{b}}$ School of Pharmacy, Faculty of Medical Sciences, Newcastle University, Newcastle upon Tyne, NE1 7RU, United Kingdom

* Correspondence: v.khutoryanskiy@ reading.ac.uk; Tel.: +44 (0) 1183786119

Keywords: Mucoadhesion, Rat, Stability, Fluorescence, Thiolated, POZylation 


\section{Abstract}

In this study, we synthesised thiolated silica nanoparticles using 3mercaptopropyltrimethoxysilane and functionalised them with either $5 \mathrm{kDa}$ methoxy polyethylene glycol maleimide (PEG) or $5 \mathrm{kDa}$ alkyne-terminated poly(2-ethyl-2-oxazoline) (POZ). The main objectives of this study are to investigate the effects of $\mathrm{pH}$ on the size and $\xi$ potential of these nanoparticles and evaluate their mucoadhesive properties ex vivo using rat intestinal mucosa. The sizes of thiolated, PEGylated and POZylated silica nanoparticles were $53 \pm 1,68 \pm 1$ and $59 \pm 1 \mathrm{~nm}$, respectively. The size of both thiolated and POZylated nanoparticles significantly increased at $\mathrm{pH} \leq 2$, whereas no size change was observed at $\mathrm{pH}$ 2.5-9 for both these two types of nanoparticles. On the other hand, the size of PEGylated nanoparticles did not change over the studied $\mathrm{pH}$ range (1.5-9). Moreover, thiolated nanoparticles were more mucoadhesive in the rat small intestine than both PEGylated and POZylated nanoparticles. After 12 cycles of washing (with a total of $20 \mathrm{~mL}$ of phosphate buffer solution $\mathrm{pH}$ 6.8), a significantly greater amount of thiolated nanoparticles remained on the intestinal mucosa than FITC-dextran (non-mucoadhesive polymer, $\mathrm{p}<0.005$ ) and both PEGylated and POZylated nanoparticles ( $\mathrm{p}<0.05$ both). However, both PEGylated and POZylated nanoparticles showed similar retention to FITC-dextran ( $p>0.1$ for both). Thus, this study indicates that thiolated nanoparticles are mucoadhesive, whereas PEGylated and POZylated nanoparticles are non-mucoadhesive in the ex vivo rat intestinal mucosa model. Each of these nanoparticles has potential applications in mucosal drug delivery.

\section{Introduction}

Oral drug delivery is the preferred administration route for most drugs, as it has several advantages over other routes, including better patient adherence (especially for chronic diseases) and possibilities for flexible dosing (Date et al., 2016). In addition, oral dosage forms generally cost less to manufacture than other formulations (e.g. injectables, eye drops and inhalators) as they do not require sterilisation (Yun et al., 2013; Date et al., 2016) or use of complex delivery devices. However, about $70 \%$ of new drugs do not reach pre-clinical development due to low bioavailability resulting from poor oral absorption (Gao et al., 2013). Drug absorption in the gastrointestinal tract (GIT) is hampered by a number of physiological barriers, including the mucus, the harsh $\mathrm{pH}$ and digestive environment of the GIT, tight junctions, epithelial cells and sub-epithelial tissues (Lundquist \& Artursson, 2016). 
Mucus is a viscous gel secreted by goblet cells, which are found in various organs, including the eye (Kessler \& Dartt, 1994), the GIT (Deplancke \& Gaskins, 2001) and the respiratory tract (Spicer et al., 1983). It consists mainly of water ( 95\%), alongside cross-linked and entangled mucin fibres, lipids, proteins, salts, cellular debris and bacteria (Moghissi et al., 1960; Bansil \& Turner, 2006; Johansson et al., 2011; Leal et al., 2017)). Mucus can be targeted using mucoadhesive drug delivery systems that adhere to this layer to prolong the residence time of the dosage forms, leading to sustained release of the loaded drugs and enhanced bioavailability compared to conventional non-mucoadhesive formulations (Bernkop-Schnürch, 2005; Khutoryanskiy, 2011).

Several types of nanoparticles have been shown to have great potential as drug delivery systems (Nguyen et al., 2016; Hu et al., 2017; Davoudi et al., 2018). Siew et al. (2012) developed nanoparticles based on mucoadhesive quaternary ammonium palmitoyl glycol chitosan. These nanoparticles enhanced the oral absorption of both hydrophilic (ranitidine) and hydrophobic drugs (griseofulvin and cyclosporine A). Bernkop-Schnürch and co-workers have developed thiolated polymers and shown their potential in the design of mucoadhesive nanoparticulate drug delivery systems (Dünnhaupt et al., 2011; Bonengel \& Bernkop-Schnürch, 2014). Prego et al. (2006) designed chitosan nanocapsules to enhance the absorption of salmon calcitonin from intestine and prolong its action as a result of their mucoadhesive properties and strong interaction with the intestinal mucous membranes.

Previously, Khutoryanskiy et al. have developed thiolated silica nanoparticles and demonstrated their mucoadhesive properties on ocular (Irmukhametova et al., 2011) and urinary bladder mucosa (Mun et al., 2016). They also demonstrated that these nanoparticles could be easily functionalised via fluorescent labelling, PEGylation and POZylation (introduction of polyethylene glycols and polyoxazolines, respectively) (Irmukhametova et al., 2011; Irmukhametova et al., 2012; Mun et al., 2014a; Mansfield et al., 2015; Mansfield et al., 2016). PEGylation of thiolated silica nanoparticles was found to reduce the retention of thiolated silica nanoparticles on the ocular (Irmukhametova et al., 2011) and urinary bladder mucosal surfaces (Mun et al., 2016). More recently, Mansfield et al. (2015; 2016) demonstrated that POZylation of these nanoparticles could enhance their penetration into porcine gastric mucosa.

Clearly, the nature of the adhesion between two surfaces (here, the nanoparticles and the mucous membrane) is highly dependent on the properties of both (Smart, 2005; Varum et al., 
2010; Khutoryanskiy, 2011). For example, we previously showed that thiolated and PEGylated silica nanoparticles were less retentive on the ocular surface compared to the urinary bladder mucosal surface (Irmukhametova et al., 2011; Mun et al., 2016). This difference in retention might be due to the rougher structure of the latter resulting in an increased contact area (Irmukhametova et al., 2011; Khutoryanskiy, 2011; Mun et al., 2016). The mucoadhesion of POZylated silica nanoparticles has never been studied previously. Therefore, in the present work, we sought to investigate the retentive properties of these silica nanoparticles in the rat intestinal mucosa. We further analysed the physicochemical properties of these nanoparticles, particularly their $\mathrm{pH}$-stability profiles.

\section{Materials and methods}

\subsection{Materials}

3-mercaptopropyltrimethoxysilane (MPTS), maleimide terminated methoxy poly(ethylene glycol) (PEG) $5 \mathrm{kDa}$, alkyne terminated poly(2-ethyl-2-oxazoline) (POZ) $5 \mathrm{kDa}$ (polydispersity index, PDI $\leq 1.2$ ), 5,5'-dithiobis (2-nitrobenzoic acid) (DTNB), fluorescein isothiocyanate (FITC), medium molecular weight chitosan (degree of acetylation 26.1\%, 124 $\mathrm{kDa}$ ), FITC-dextran (3.5-5 kDa) and triethyl amine (TEA) were purchased from Sigma-Aldrich (Gillingham, UK). FITC-chitosan was made in house (Symonds et al., 2016a). Fluorescein-Omethacrylate (FMA), dimethyl sulfoxide (DMSO) and $\mathrm{NaOH}$ were purchased from Fisher Scientific (UK). Dialysis membrane with molecular cut-off 12-14 kDa was purchased from Medicell International Ltd., UK.

\subsection{Synthesis of thiolated silica nanoparticles}

Thiolated silica nanoparticles were synthesised according to a previously published method (Irmukhametova et al., 2011). In brief, $20 \mathrm{~mL}$ DMSO and $0.5 \mathrm{~mL}$ of $0.5 \mathrm{M} \mathrm{NaOH}$ solution were added to $0.75 \mathrm{~mL}$ MPTS. The mixture was continuously stirred and aerated for 24 hours at room temperature. Next, the nanoparticle suspensions were dialysed against deionised water $(4 \mathrm{~L}, 8$ changes of water over 2 days) using the dialysis membrane. The purified nanoparticle suspensions were refrigerated at $4{ }^{\circ} \mathrm{C}$ until use.

\subsection{Synthesis of PEGylated and POZylated silica nanoparticles}

Thiolated silica nanoparticles were functionalised using two different polymers; PEG and POZ. To synthesise PEGylated nanoparticles, $100 \mathrm{mg}$ PEG was added to $10 \mathrm{~mL}$ thiolated 
nanoparticle suspension and the mixture was stirred for 24 hours at room temperature. To synthesise POZylated particles, $5 \mathrm{~mL}$ of thiolated nanoparticle suspension was diluted with 5 $\mathrm{mL}$ DMSO and then $100 \mathrm{mg}$ POZ was added to the diluted thiolated nanoparticles. To this mixture, $200 \mu \mathrm{L}$ TEA was added to enhance the thiol-yne click reaction (Mansfield et al., 2015). The reaction mixture was left for 24 hours with continuous stirring. The nanoparticles were purified by dialysis as described in section 2.2 and refrigerated at $4{ }^{\circ} \mathrm{C}$ until use.

\subsection{Fluorescent labelling of nanoparticles}

Each of the thiolated, PEGylated and POZylated silica nanoparticle suspensions ( $5 \mathrm{~mL})$ were diluted with DMSO $(5 \mathrm{~mL})$. To this, $2 \mathrm{~mL}(3.59 \mu \mathrm{mol})$ of $1.8 \mathrm{mM}$ fluorescein-O-methacrylate solution (in 1:1 deionised water: ethanol) and $200 \mu \mathrm{L}$ TEA was added. The reaction mixture was stirred in the dark for 24 hours. Subsequently, the fluorescently labelled nanoparticles were purified (again in the dark) as described in section 2.2.

\subsection{Characterisation of nanoparticles}

The size and $\xi$-potential of the nanoparticles were measured using Zetasizer Nano-ZS (Malvern, UK). For the size measurements, the samples were diluted 1:100 with ultrapure water before analysis. A refractive index of 1.475 and an absorbance of 0.1 were used for all measurements. Measurements were conducted in triplicate for 10 seconds per run, with 12 runs per reading at $25^{\circ} \mathrm{C}$. $\xi$-potential values were measured using DTS-1070 folded capillary tube cuvettes (Malvern, UK). Samples were measured using 3 repeats of 20 sub-runs per reading. At least 3 samples were measured and processed using the Smoluchowski model $(\mathrm{F} \kappa \mathrm{a}=1.50)$. Transmission electron microscopy (TEM) was conducted using a JEM-2100 PLUS Electron Microscope (JEOL, USA) at an accelerating voltage of $200 \mathrm{kV}$. Three drops of nanoparticle suspensions were placed on a carbon-coated copper grid and left for 1 minute before being loaded into the instrument. The morphology of the nanoparticles was investigated without any staining.

\subsection{Determination of thiol content}

Ellman's assay was used to quantify the free thiol groups available on the surface of the nanoparticles. Initially, the nanoparticles were lyophilised using the Heto Power Dry LL 3000 freeze-drier (Thermo Electron Corporation). The nanoparticles ( $3 \mathrm{mg}$ ) were suspended in 10 $\mathrm{mL}$ phosphate buffer solution ( $0.5 \mathrm{M}, \mathrm{pH}$ 8). Then, $0.5 \mathrm{~mL}$ aliquots of nanoparticle suspension was reacted with $0.5 \mathrm{~mL}$ DTNB $(0.3 \mathrm{mg} / \mathrm{mL})$ in the dark for 2 hours. Next, the product was 
centrifuged for 10 minutes at $13000 \mathrm{rpm}$ (Sanyo, Micro Centaur, UK) and $200 \mu \mathrm{L}$ aliquots of supernatant was loaded into a 96 well-plate. The light absorbance was measured at $420 \mathrm{~nm}$ using an Epoch microplate reader (BioTek Instruments, Inc.). In order to obtain a calibration curve, serial solutions of L-cysteine $\mathrm{HCl}$ over the concentration range of 0.004 to 0.634 $\mu \mathrm{mol} / \mathrm{mL}$ were prepared and reacted with DTNB under the same conditions as the nanoparticles. Phosphate buffer solution ( $\mathrm{pH}$ 8) was used as the blank control. Finally, the amount of free thiols per gram of the particles was calculated.

\subsection{FTIR spectroscopy}

FTIR spectra of the freeze-dried nanoparticles were recorded using a Spectrum 100 FTIR spectrophotometer (Perkin-Elmer, UK). The spectra were collected from an average of 4 scans, with a resolution of $4 \mathrm{~cm}^{-1}$ over the range of $4000-650 \mathrm{~cm}^{-1}$.

\subsection{Thermogravimetric analysis (TGA)}

Freeze-dried samples were analysed for all three types of silica nanoparticles using the Q50 thermogravimetric analyser (TA Instruments, UK) equipped with nitrogen to provide an inert environment. The instrument was zeroed against an empty differential scanning calorimetry aluminium pan. The samples were placed in an aluminium pan and then in a platinum TGA pan and loaded into the instrument. The initial temperature was set at $35{ }^{\circ} \mathrm{C}$ and the thermal decomposition of the samples was studied between 35 and $500{ }^{\circ} \mathrm{C}$, at $5{ }^{\circ} \mathrm{C} /$ minute heating rate.

\subsection{Fluorescence spectroscopy}

Fluorescently labelled nanoparticle suspensions were diluted (1:400-1:12,800) with PBS (pH 7.4). The fluorescence emission spectra were measured between 500 - $700 \mathrm{~nm}$ (emission wavelength) using a fluorescence spectrometer (Cary Eclipse, Varian Inc., US) at $490 \mathrm{~nm}$ excitation wavelength.

\subsection{0 pH-stability study}

Unlabelled nanoparticle suspensions were diluted with ultrapure water (1:100, $60 \mathrm{~mL}$ and 40 $\mathrm{mL}$ of the diluted nanoparticle suspensions were prepared for experiments of decreasing and increasing $\mathrm{pH}$, respectively). The $\mathrm{pH}$ was adjusted using $1 \mathrm{M} \mathrm{HCl}$ or $1 \mathrm{M} \mathrm{NaOH}$ solutions. Then at each $\mathrm{pH}$ point, the size and $\xi$-potential of the nanoparticles were measured as described in section 2.5. 


\subsection{Determination of minimum detection limit of nanoparticles by fluorescence microscopy}

The minimum detection limit of the nanoparticles was determined by first measuring the concentration of fluorescently labelled thiolated nanoparticles by gravimetry $(1.15 \pm 0.20$ $\mathrm{mg} / \mathrm{mL} ; n=3$ ). This was performed by placing $1 \mathrm{~mL}$ of thiolated nanoparticles in a glass container and heating on a hotplate, initially at $100{ }^{\circ} \mathrm{C}$ for a few minutes, then at $75{ }^{\circ} \mathrm{C}$. The sample was weighed periodically and re-heated until a constant weight was obtained, indicating complete drying of the nanoparticles. Then, serial suspensions of fluorescently labelled thiolated nanoparticles were prepared in deionised water over the concentration range of 0.004 $0.533 \mathrm{mg} / \mathrm{mL}$. Diluted thiolated nanoparticle suspensions $(20 \mu \mathrm{L})$ were applied on $0.8 \times 1.2$ $\mathrm{cm}^{2}$ rat intestinal mucosa (tissues sample was used within 10 minutes of thawing). Fluorescent images were recorded using a fluorescence microscope (Leica Microsystems, UK) at $160 \mathrm{~ms}$ exposure time. The images were then analysed by ImageJ (National Institutes of Health, USA) and the coefficient of determination $\left(R^{2}\right)$ was calculated.

\subsection{Mucoadhesion study}

Fluorescence microscopy was used to investigate the mucoadhesion of the synthesised nanoparticles. A Leica MZ10F fluorescence microscope (Leica Microsystems, UK) with ETGFP filter, maximum excitation light intensity and $160 \mathrm{~ms}$ exposure time was used (in case of FITC-chitosan, however, $211 \mathrm{~ms}$ exposure time was used to compensate its poor fluorescence emission). In this study, freshly isolated small intestinal tissue from healthy 3 to 4 month-old Sprague Dawley female rats was used. It should be noted that these rats were used in a completely different study by our colleagues and were sacrificed for the purpose of that work; we have simply harvested small intestinal tissues from these animals. The intestines were cut open to approximately $0.8 \times 1.2 \mathrm{~cm}^{2}$ and placed on a microscope slide. Fluorescence micrographs of the mucosal surface were taken as a background fluorescence intensity. Thereafter, a $20 \mu \mathrm{L}$ suspension of fluorescently labelled nanoparticles was placed on the intestinal mucosal surface and incubated for 5 minutes, before fluorescence micrographs were recorded again. The tissues were transferred to a sloped channel and washed with $1 \mathrm{~mL}$ or 5 $\mathrm{mL}$ of phosphate buffer solution $\left(100 \mathrm{mM}, \mathrm{pH} 6.8,37 \pm 1{ }^{\circ} \mathrm{C}\right)$ for the total of 13 cycles. Fluorescence micrographs were recorded sequentially after each wash cycle. The images were analysed using the ImageJ software and normalised according to the following equation:

Fluorescence intensity $=\frac{I-I_{b}}{I_{o}-I_{b}} \times 100 \quad$ Equation (1), 
where, $I$ is the fluorescence intensity of a given tissue sample nanoparticles after each wash cycle, $I_{\mathrm{b}}$ is the background fluorescence intensity of that tissue sample, and $I_{\mathrm{o}}$ is the initial fluorescence intensity of that sample (i.e. following nanoparticles incubation but preceding any wash cycle). Each tissue sample provided its own control for background fluorescence intensity. The experiments were performed in triplicate using tissues obtained from 3-4 rats, and the order in which each nanoparticle type was studied in any individual experiment was randomised. FITC-chitosan $(1 \mathrm{mg} / \mathrm{mL}$ in $0.1 \mathrm{M}$ acetic acid) and FITC-dextran $(1 \mathrm{mg} / \mathrm{mL}$ in deionised water) were used as positive and negative controls, respectively.

In addition, $\mathrm{WO}_{50}$ (Mun et al., 2016) and $\mathrm{WO}_{70}$ values were quantified. These values represent the amount of phosphate buffer solution necessary to attenuate the fluorescence intensity on the mucosal surface by $50 \%$ and $70 \%$, respectively. The values were calculated by extrapolation of the average wash-off data using 6th order polynomial fitting and Wolfram Alpha (a computational knowledge engine).

\subsection{Statistical analysis}

Unless otherwise stated, all measurements were collected in triplicate and the data are expressed as mean \pm standard deviation (SD). The data were analysed using the SPSS Statistics 21 program (IBM, US). The statistical significance of any difference between groups was determined using one-way analysis of variance (ANOVA) with the least significant difference (LSD) post-hoc test. Differences were considered statistically significant at $p<0.05$.

\section{Results and discussion}

\subsection{Nanoparticles synthesis and characterisation}

Thiolated nanoparticles were synthesised via hydrolysis and subsequent condensation of the methoxysilane groups of MPTS, forming a cross-linked nanoparticle structure through Si-OSi and disulfide bonds. All nanoparticle samples exhibited acceptable PDI (PDI $<0.2$, as determined by DLS), with a single peak around the z-average of the particles (Fig. 1 and Table 1). Particles with PDI $<0.05$ are highly monodisperse but rarely seen unless with standard nanoparticles. Nanoparticles with PDI $>0.7$ have generally very broad size distribution and not recommended to be sized using DLS technique. Thus, lower PDI indicates higher homogeneity of the nanoparticles and vice versa (Malvern Instruments Ltd., 2011). The particle size 
increased in the order of thiolated < POZylated < PEGylated nanoparticles (Fig. 1 and Table $1)$.

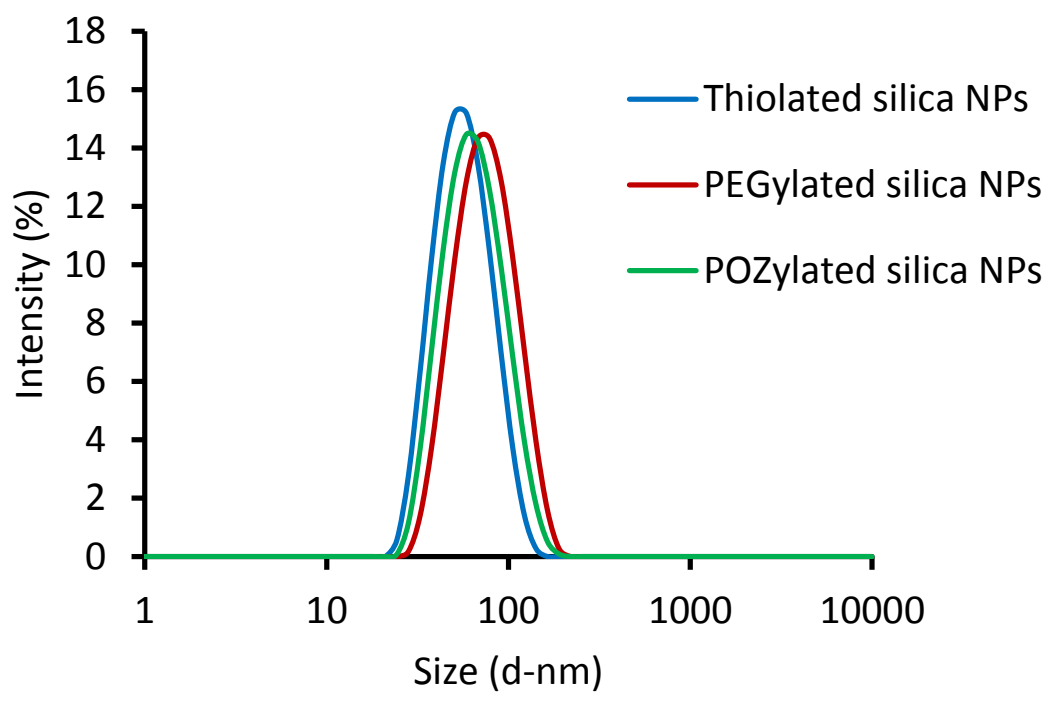

Fig. 1. Exemplar dynamic light scattering size distributions of thiolated, PEGylated and POZylated silica nanoparticles.

\section{Table 1}

Characteristics of unlabelled thiolated, PEGylated and POZylated silica nanoparticles (mean $\pm \mathrm{SD}, \mathrm{n}=3$ ); for size and $\xi$-potential measurement, the nanoparticle suspensions were diluted with ultrapure water $(1: 100)$.

\begin{tabular}{llllll}
\hline $\begin{array}{l}\text { Types of } \\
\text { particles }\end{array}$ & Size $(\mathrm{nm})$ & PDI & $\begin{array}{l}\xi \text {-potential } \\
(\mathrm{mV})\end{array}$ & $\begin{array}{l}\text { Conc. }^{\mathrm{a}} \\
(\mathrm{mg} / \mathrm{mL})\end{array}$ & $\begin{array}{l}\text { Free thiol } \\
\text { content } \\
(\mu \mathrm{mol} / \mathrm{g})\end{array}$ \\
\hline Thiolated & $53 \pm 1$ & $0.118 \pm 0.012$ & $-42 \pm 2$ & $6 \pm 2$ & $368 \pm 11$ \\
PEGylated & $68 \pm 1$ & $0.128 \pm 0.002$ & $-22 \pm 3$ & $13 \pm 1$ & $175 \pm 2$ \\
POZylated & $59 \pm 1$ & $0.134 \pm 0.014$ & $-31 \pm 4$ & $5 \pm 1$ & $53 \pm 18$ \\
\hline
\end{tabular}

${ }^{\mathrm{a}}$ measured gravimetrically after freeze drying nanoparticle dispersions

The difference in particle size between each pair of nanoparticle type was statistically significant $(p<0.05)$. The larger PEGylated and POZylated nanoparticles indicate successful 
grafting of the polymers onto the thiolated nanoparticles core. PEG $5 \mathrm{kDa}$ contains 113 repeating units of ethylene glycol $(\mathrm{Mr}=44)$, whereas POZ $5 \mathrm{kDa}$ consists of 50 repeating units of 2-ethyl-2-oxazoline ( $\mathrm{Mr}=99)$. This means that the PEG chains were longer than the POZ chains, resulting in a thicker shell and thus larger particles (Mansfield, 2016). Another possible reason for the size discrepancy between PEG and POZ nanoparticles is related to the different spatial arrangement of PEG and POZ around the core nanoparticles. Typically, PEG molecules form long-brush or mushroom configurations. The former happens when the density of PEG on the surface of nanoparticles is high, while the latter occurs in cases of low surface coverage (Owens \& Peppas, 2006). In contrast, POZ forms star-shaped structures (Rossegger et al., 2013). Also, it can be hypothesised that different polymer chain folding and relaxation behaviour in water may also contribute to the difference in the size of the corresponding nanoparticles.

The concentration of the nanoparticles was estimated using freeze drying by determining the dry weight of the nanoparticles in $1 \mathrm{~mL}$ of suspensions. The results showed that PEGylated nanoparticles were more concentrated than both thiolated and POZylated nanoparticles (Table 1). These concentration estimates were used to quantify the free thiol contents of the nanoparticles.

In this study, all three types of silica nanoparticles (thiolated, PEGylated and POZylated) were labelled with a fluorescent marker, by reacting them with FMA (Fig. S1 and S2). The fluorescent molecules were covalently bound to the nanoparticles via thiol-ene click reaction between the thiol groups of the nanoparticles and the vinyl groups of FMA. Fluorescent labelling enabled facile quantification of the nanoparticles on the surface of rat intestinal mucosa, with virtually no change in the particle size (see Table 1 and 2). In addition, no signs of aggregation or flocculation was observed for both fluorescently labelled and unlabelled particles during the study (ca. 6 months). This indicates that FMA imparted a characteristic fluorescent signal to the particles without impairing the colloidal stability of the suspensions. 
Ellman's assay showed a statistically significant difference in free thiol content among all three types of nanoparticles $(p<0.05)$. Free thiol content decreased in the order of thiolated > PEGylated > POZylated nanoparticles (Table 1). This result indicates successful functionalisation of both PEGylated and POZylated nanoparticles, since PEGylation and POZylation were expected to replace free thiol groups with the corresponding polymers. The calibration curve used in the Ellmans's assay is shown in Fig. S3.

\section{Table 2}

Properties of fluorescently labelled thiolated, PEGylated and POZylated silica nanoparticles (for fluorescence measurement, nanoparticles were diluted with PBS pH 7.4, 1:1,600), mean \pm $\mathrm{SD}, \mathrm{n}=3$.

\begin{tabular}{ccccc}
\hline $\begin{array}{c}\text { Types of } \\
\text { particles }\end{array}$ & Size $(\mathrm{nm})$ & PDI & $\begin{array}{c}\text { Max. emission } \\
(\mathrm{nm})\end{array}$ & $\begin{array}{c}\text { Max. intensity } \\
(\text { a.u. })\end{array}$ \\
\hline Thiolated & $51 \pm 1$ & $0.200 \pm 0.018$ & 512 & $411 \pm 36$ \\
PEGylated & $71 \pm 2$ & $0.106 \pm 0.020$ & 512 & $233 \pm 14$ \\
POZylated & $61 \pm 3$ & $0.117 \pm 0.027$ & 512 & $405 \pm 38$ \\
\hline
\end{tabular}

In agreement with our previous study (Irmukhametova et al., 2012), TEM results show that thiolated nanoparticles aggregated and formed 'necklaces'(Fig 2). A possible reason for the aggregation is disulfide bond formation between the nanoparticles, facilitated by drying during TEM sample preparation. In contrast, TEM images of PEGylated and POZylated nanoparticles did not show any signs of aggregation. This observation, together with DLS data, confirm the presence of primarily non-aggregated PEGylated and POZylated particles in the samples. Thus, although the $\xi$-potential of both PEGylated and POZylated nanoparticles had significantly decreased compared to the parent thiolated nanoparticles (Table 1), their colloidal stability seemed to have dramatically improved. In addition to the overall reduction in reactive thiol groups in PEGylated and POZylated nanoparticles, steric hindrance (i.e. shielding of remaining thiol groups by the hydrophilic polymeric shells on the particle surface) is believed to be a major contributing factor to the improvement of colloidal stability. 

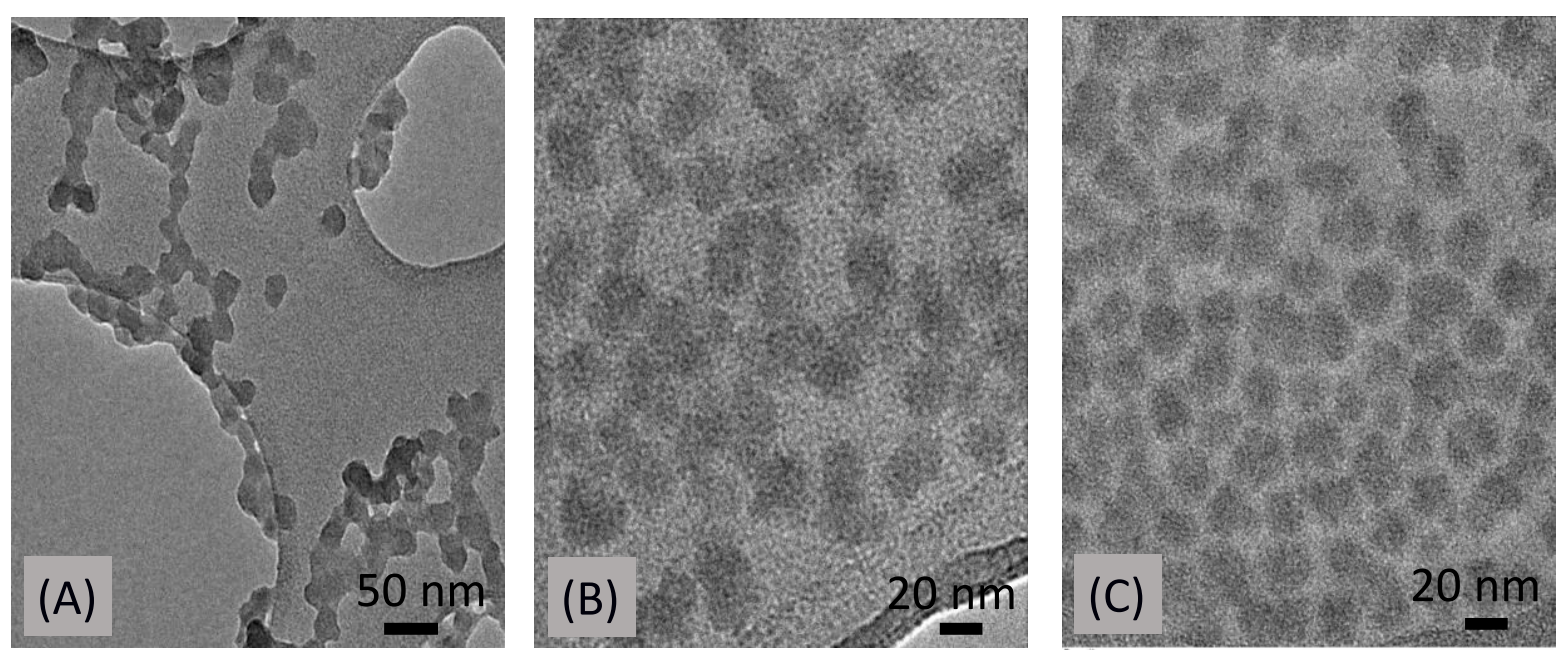

Fig. 2. TEM images of unlabelled thiolated (A), PEGylated (B) and POZylated (C) silica nanoparticles. Thiolated nanoparticles formed necklace-like structures, whereas both PEGylated and POZylated nanoparticles were mainly individually distributed.

FTIR spectra and TGA thermograms are shown in Fig. S4 and S5, respectively. These results were in agreement with our previous studies (Irmukhametova et al., 2012; Mansfield et al., 2015; Mansfield et al., 2016).

As shown in Fig. S1, S2 and Table 2, PEGylated nanoparticles fluoresced less intensely $(\mathrm{p}<$ $0.05)$ than thiolated particles. This is most likely due to the screening effect of non-fluorescent PEG on the surface of these PEGylated nanoparticles. However, POZylated nanoparticles showed similar fluorescence intensity compared to thiolated nanoparticles $(\mathrm{p}>0.05)$, which was significantly higher $(\mathrm{p}<0.05)$ than that of PEGylated nanoparticles. The shorter POZ chain length (50 repeating units) compared to PEG (113 repeating units) (Mansfield et al., 2015) potentially led to less fluorescence screening resulting in a higher fluorescence signal in the POZylated nanoparticles.

\section{2.pH-stability study}

The dosage form administered orally is expected to path through regions of gastrointestinal tract with different $\mathrm{pHs}$. The $\mathrm{pH}$ along the GIT ranges from around 2 in the stomach, to 6-7 in the small intestine and about 8 in the colon. We therefore evaluated the stability of thiolated, PEGylated and POZylated silica nanoparticles over the $\mathrm{pH}$ range of 1.5-9, in terms of particle size, PDI and $\xi$-potential. The initial pHs of the diluted thiolated, PEGylated and POZylated silica nanoparticle suspensions were 5.93, 5.67 and 6.08, respectively. Thiolated and 
POZylated nanoparticles were stable over the $\mathrm{pH}$ range of 2.5-9 as no change in their z-average size was observed. However, they aggregated at $\mathrm{pH} \leq 2$, resulting in a significant increase in particle size (z-average: $4.29 \pm 0.78 \mu \mathrm{m}$ for thiolated and $6.84 \pm 0.52 \mu \mathrm{m}$ for POZylated nanoparticles) and increase in polydispersity (Fig. 4-A, B and C). This aggregation was irreversible as particle size was not reduced when higher $\mathrm{pH}$ was restored. The aggregation of the particles at $\mathrm{pH}<2$ could be a result of $\mathrm{Si}-\mathrm{O}-\mathrm{Si}$ covalent bond formation between the nanoparticles, owing to the availability of $\equiv \mathrm{SiOH}$ groups on the surface of the particles (Irmukhametova et al., 2012). The instability of thiolated and POZylated nanoparticles at pH $\leq 2$ may potentially limit their application for oral drug delivery; however, this limitation could potentially be overcome by encapsulating these particles into a vehicle protected with $\mathrm{pH}$ sensitive enteric coating such as Eudragits or their complexes with other polymers (Moustafine et al, 2011; Mustafin, 2011). In contrast, PEGylated nanoparticles were stable over the entire $\mathrm{pH}$ range investigated (1.5-9), as they showed neither aggregation nor dissociation.

(A)

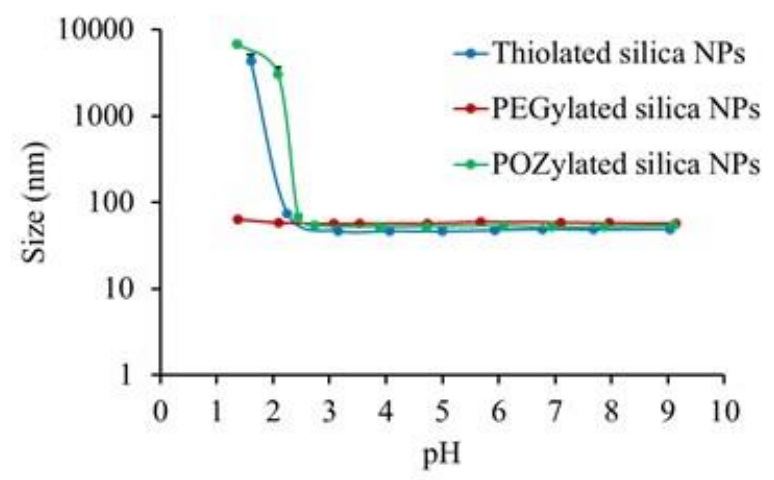

(C)

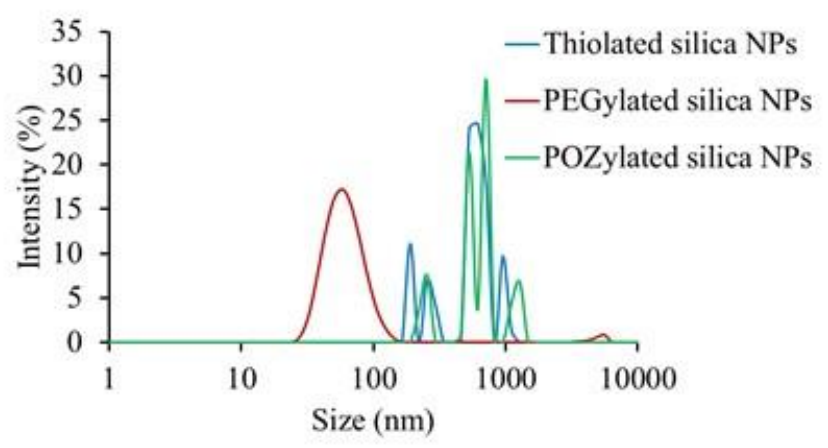

(B)

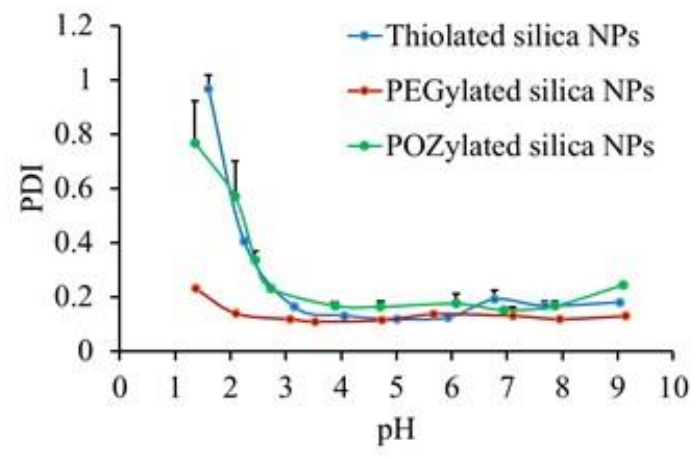

(D)

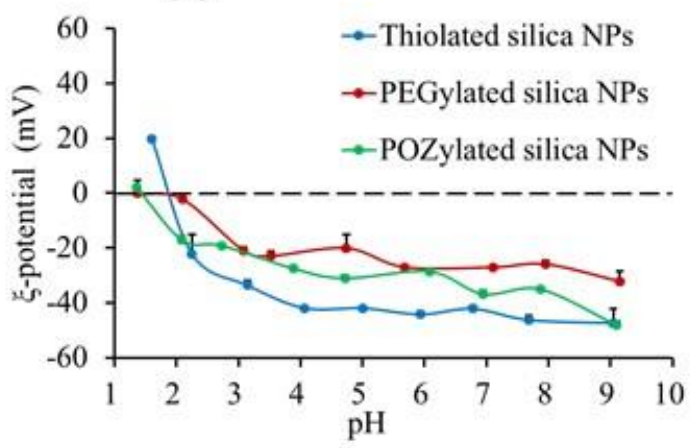


Fig. 4. DLS average size (A), PDI (B) at different $\mathrm{pH}$ environments, particle size distribution at $\mathrm{pH} 1.5(\mathrm{C})$ and $\xi$-potential of thiolated, PEGylated and POZylated silica nanoparticles in different $\mathrm{pH}$ environments $(\mathrm{D})$, mean $\pm \mathrm{SD}, \mathrm{n}=3$. No error bars shown in $(\mathrm{C})$.

The $\xi$-potential of the nanoparticles was lower at low pH than at high pH (Fig. 4-D). At pH 1.5 the thiolated nanoparticles, however, had a more positive value $(20 \pm 1.0 \mathrm{mV})$ compared to PEGylated and POZylated nanoparticles $(-0.2 \pm 5.0 \mathrm{mV}$ and $2.0 \pm 1.0 \mathrm{mV}$, respectively). The reduction in the $\xi$-potential could be due to the protonation of the negatively charged $\mathrm{SiO}^{-}$and $\mathrm{S}^{-}$ions, resulting in neutral $\mathrm{SiOH}$ or $\mathrm{SH}$ (Dyab, 2012). Interestingly, at low $\mathrm{pH}(\sim 1.5)$ both PEGylated and POZylated nanoparticles showed a lower $\xi$-potential than thiolated nanoparticles. This could be explained by the fact that a significant number of SH groups were already substituted with PEG or POZ.

\subsection{Ex vivo mucoadhesion study}

In order to conduct the evaluation of mucoadhesive properties of the nanoparticles, the minimal detectable concentration of fluorescently labelled particles on the surface of rat intestinal mucosa was determined. The fluorescence of labelled particles was detectable even upon dilution by nearly 60-fold (Fig. S6). This result is also important for future studies involving the monitoring of these fluorescent nanoparticles in the GIT following oral administration, as the particles are likely to be diluted by GI fluids. As shown in Fig. S6, there is a linear relationship $\left(\mathrm{R}^{2}=0.9968\right)$ between the concentration of thiolated nanoparticles and their fluorescence intensity.

Using a wash-off technique (Ranga Rao \& Buri, 1989; Nielsen et al., 1998) in combination with fluorescence microscopy, the retention of fluorescently labelled nanoparticles on the surface of rat intestinal mucosa was studied. This technique has been previously used to study the retention of various materials on different surfaces including animal tissues and plant leaves (Irmukhametova et al., 2011; Štorha et al., 2013; Cook et al., 2015; Mun et al., 2016; Symonds et al., 2016b; Tonglairoum et al., 2016).

Samples were applied on the surface of rat intestinal mucosa and then washed with phosphate buffer pH 6.8 over several cycles. The fluorescence intensity on the mucosa was monitored following each wash cycle (Fig. 5). Based on the linear relationship established between nanoparticles concentration and fluorescence intensity (Fig. S6), the amount of fluorescent 
nanoparticles remaining on the tissue was estimated. As both fluorophores (FMA and FITC) were covalently bound to the materials used in this study (nanoparticles, chitosan and dextran), it can be assumed that the fluorescence intensity was directly proportional to the percentage of materials retained on the mucosal surface. However, this method is likely to underestimate the amount of labelled nanoparticles/macromolecules as they are diluted by phosphate buffer solution during the wash cycles. In addition, a portion of the nanoparticles/macromolecules may diffuse into the mucus layer, resulting in attenuation or even obliteration of the fluorescent signal. However, both these factors were excluded in this particular study and the $\%$ remaining of all nanoparticles, FITC-chitosan and FITC-dextran (positive and negative controls) were calculated as described in section 2.11. 
FITC-chitosan
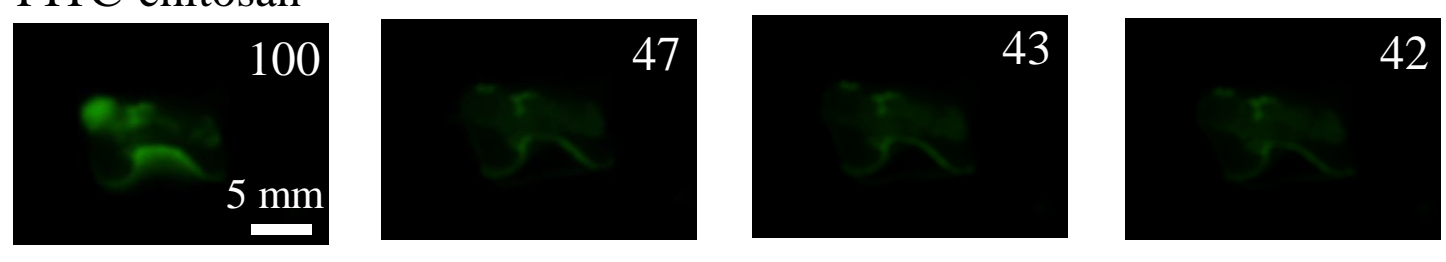

\section{Thiolated silica NPs}
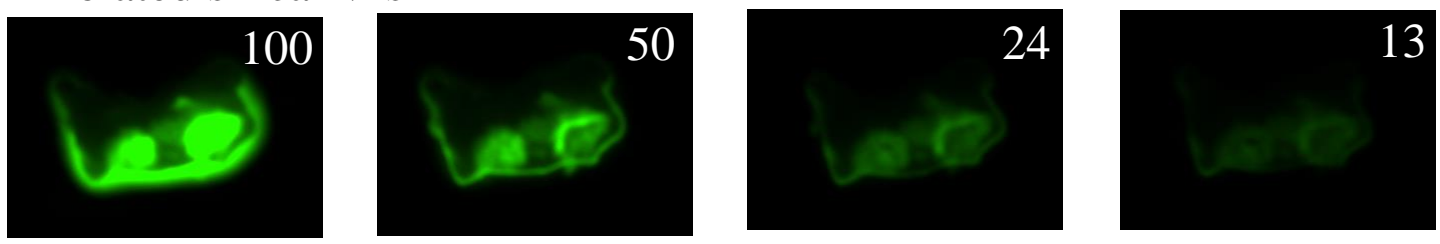

PEGylated silica NPs
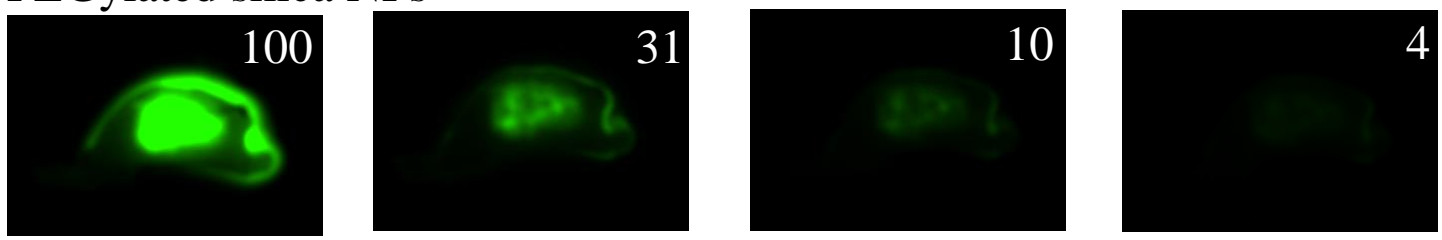

POZylated silica NPs
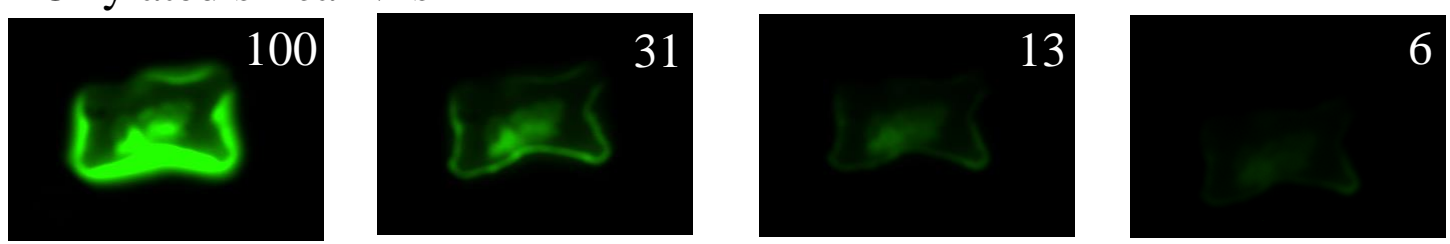

FITC-dextran
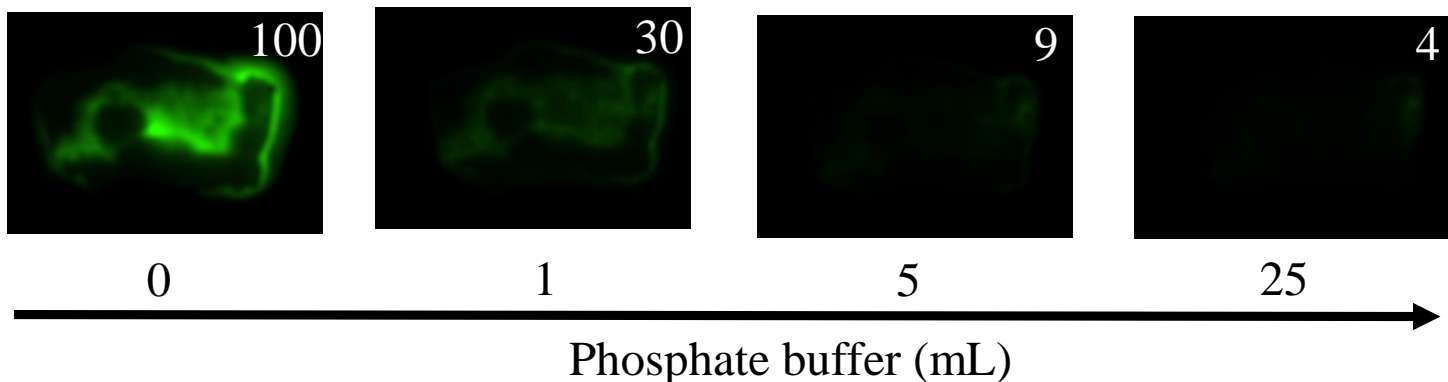

Fig. 5. Exemplar fluorescence microphotographs showing retention of FITC-chitosan (positive control used as a mucoadhesive material), thiolated, PEGylated and POZylated silica nanoparticles and FITC-dextran (negative control used as a non-mucoadhesive material) on rat intestinal mucosa following wash-out with phosphate buffer solution at $\mathrm{pH}$ 6.8. The values of the normalised fluorescence intensity were inserted into the top right corners of the representative images (a.u.). After washing the fluorescent nanoparticles by certain amounts of phosphate buffer solution, the fluorescence intensity from thiolated nanoparticles was greater than for PEGylated and POZylated nanoparticles. The scale bar is $5 \mathrm{~mm}$. 
At the end of each wash cycle, the \% of thiolated nanoparticles remaining was significantly greater $(p<0.05)$ than FITC-dextran, which was used as a non-mucoadhesive material (negative control) (Fig. 6). Several studies have reported the non-mucoadhesive nature of dextran, which is likely due to its non-ionic character leading to muco-inertness instead of mucoadhesion (Khutoryanskiy, 2011; Withers et al., 2013; Mun et al., 2016). Thiolated nanoparticles also showed better retention than both PEGylated and POZylated nanoparticles $(\mathrm{p}<0.05)$ (Fig. 6).

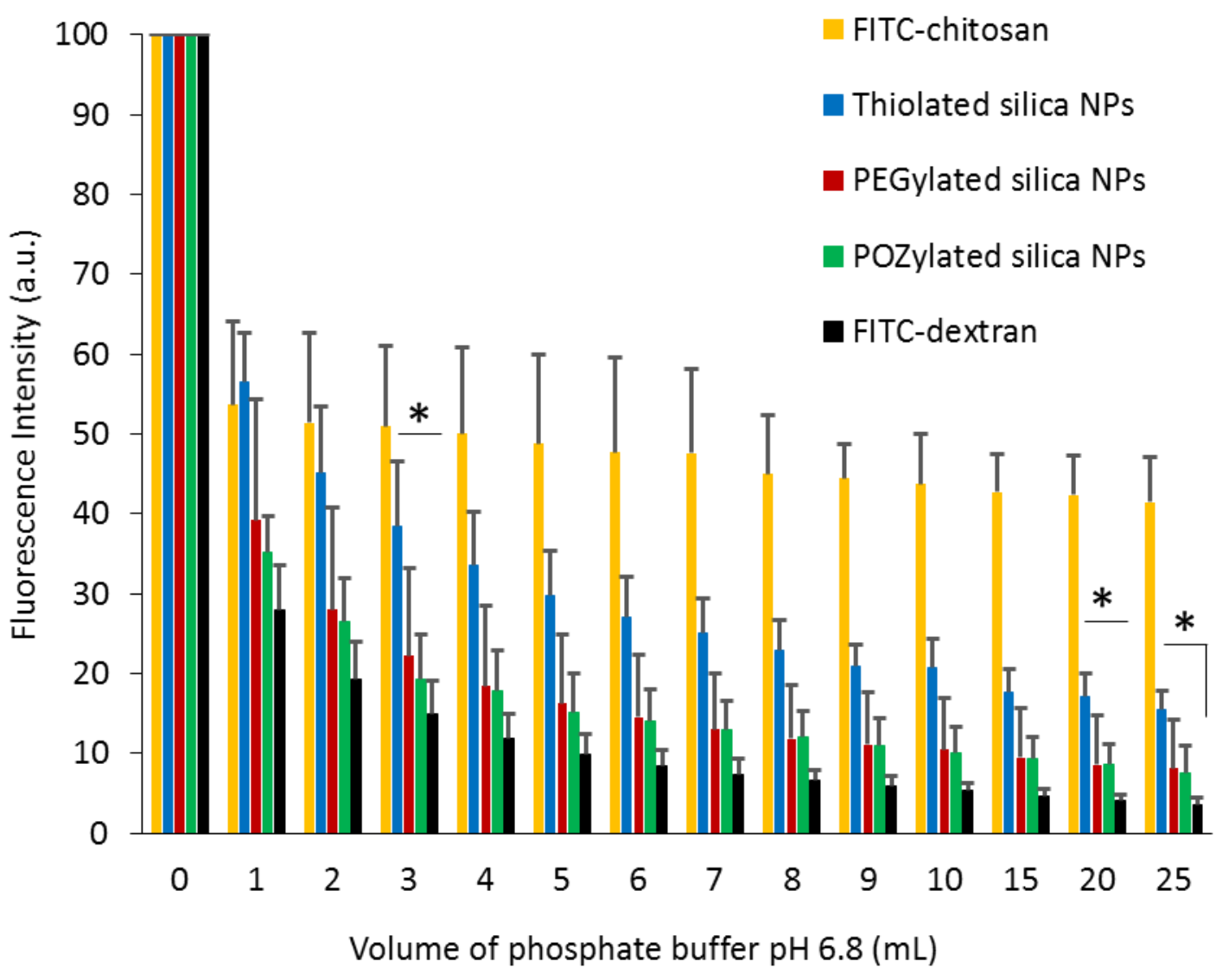

Fig. 6. Fluorescence level of rat intestinal mucosa exposed to FITC-chitosan, fluorescently-labelled thiolated, PEGylated and POZylated silica nanoparticles and FITC-dextran washed with phosphate buffer solution (100 mM, pH 6.8) (n= 3 using 3-4 rats, mean $\pm \mathrm{SD}$, “*” represents $\mathrm{p}<0.05)$. 
FITC-chitosan (positive control) showed remarkably higher retention than FITC-dextran (Fig. 6). Many studies have confirmed the adhesion of chitosan to various mucosal surfaces, including the eye, stomach and urinary bladder, owing to its cationic nature and ability to form electrostatic and hydrogen bonds with mucus components (Sogias et al., 2008; Mun et al., 2016; Tonglairoum et al., 2016). Over the first 3 wash cycles, thiolated nanoparticles showed a similar retention profile to FITC-chitosan $(p>0.05)$. However, at the end of each wash cycle, neither PEGylated nor POZylated nanoparticles showed any statistically significant difference compared to FITC-dextran $(\mathrm{p}>0.05)$. This similarity of PEGylated and POZylated nanoparticles with FITC-dextran was persistent until the end of all wash cycles. In contrast, POZylated and PEGylated nanoparticles showed significantly less mucoadhesion than FITCchitosan starting from the first and second wash cycle, respectively $(\mathrm{p}<0.05$ for both POZylated and PEGylated nanoparticles) (Fig. 6).

Mun et al. (2016) demonstrated that about $80 \%$ of thiolated silica nanoparticles, and only about $40 \%$ of PEGylated nanoparticles, remained on the surface of bladder mucosal tissues after washing with $10 \mathrm{~mL}$ artificial urine. In comparison, we found less retention for all three types of nanoparticles (thiolated: $21 \%$, PEGylated: $11 \%$, POZylated: $10 \%$ ) after washing with the same volume of the bio-relevant fluid (Fig. 6). This is because porcine bladder mucus is much thicker $(1.4 \pm 0.5 \mathrm{~mm})$ than rat small intestinal mucus $(30.6 \pm 8.8 \mu \mathrm{m})$. A thicker mucus provides deeper "interdiffusion and entanglement" between the mucoadhesive particles and the mucins. Thus, a longer contact time might be achieved and this probably makes more groups available for the formation of chemical bonds responsible for mucoadhesion (Varum et al., 2010).

In addition to the method developed by Mun et al. (2016) to determine $\mathrm{WO}_{50}$, we propose to calculate $\mathrm{WO}_{70}$ of the formulations. $\mathrm{WO}_{50}$ and $\mathrm{WO}_{70}$ represent the volume of phosphate buffer solution required to wash out $50 \%$ and $70 \%$, respectively, of the formulations from the rat intestinal mucosa. Calculation of $\mathrm{WO}_{70}$ is important as generally a greater amount of both thiolated and PEGylated nanoparticles were washed off from rat intestinal mucosa compared to urinary bladder mucosa (Mun et al., 2016). Similar differences in mucosal retention have also been reported by Irmukhametova et al. (2011), Mun et al. (2016) and Štorha et al. (2013) on bovine corneal and porcine urinary bladder tissues. A few studies reported the similarity of the thickness and the structure of the mucus of human GIT to that of porcine and showed some resemblance of the two (Varum et al., 2010, 2012). However, rat intestinal mucosa is an ex vivo model widely used to measure the oral absorption of drugs and evaluate the mucoadhesion 
and/or mucus-penetration of various drug delivery systems including nanoparticles (Lehr et al., 1992; Keely et al., 2005; Dünnhaupt et al., 2011; Merchant et al., 2014; Maisel et al., 2015; Oltra-Noguera et al., 2015; Lozoya-Agullo et al., 2016).

Therefore, using $\mathrm{WO}_{70}$ allows the comparison of different nanoparticles in terms of their washoff profiles. Table 3 shows that $\mathrm{WO}_{50}$ values for FITC-chitosan $(1.9 \mathrm{~mL})$ and thiolated nanoparticles $(1.58 \mathrm{~mL})$ are nearly double compared to those for PEGylated $(0.95 \mathrm{~mL})$ and POZylated nanoparticles $(0.88 \mathrm{~mL})$. The $\mathrm{WO}_{70}$ for FITC-chitosan could not be determined since a sizeable proportion ( $>40 \%$ ) of the formulation remained tenaciously on the intestinal mucosa withstanding the multiple washing cycles (Fig. 5 and 6). On the other hand, the $\mathrm{WO}_{70}$ for thiolated nanoparticles was approximately 3-fold higher than for PEGylated and POZylated nanoparticles. This indicates that thiolated nanoparticles resisted a greater amount of wash buffer than both PEGylated and POZylated nanoparticles. $\mathrm{WO}_{50}$ and $\mathrm{WO}_{70}$ of both PEGylated and POZylated nanoparticles were close to that of FITC-dextran denoting the nonmucoadhesive nature of these two types of nanoparticles (Table 3).

\section{Table 3}

Values of $\mathrm{WO}_{50}$ and $\mathrm{WO}_{70}$ for FITC-chitosan, thiolated, PEGylated and POZylated silica nanoparticles, as well as FITC-dextran in rat intestinal mucosa.

\begin{tabular}{ccc}
\hline Types of materials & $\mathrm{WO}_{50}(\mathrm{~mL})$ & $\mathrm{WO}_{70}(\mathrm{~mL})$ \\
\hline FITC-chitosan & 1.90 & $\mathrm{NA}$ \\
Thiolated silica nanoparticles & 1.58 & 5.26 \\
PEGylated silica nanoparticles & 0.95 & 1.73 \\
POZylated silica nanoparticles & 0.88 & 1.58 \\
FITC-dextran & 0.76 & 1.32 \\
\hline
\end{tabular}

Polynomial fitting $\left(6^{\text {th }}\right.$ order $)$ was used to calculate $\mathrm{WO}_{50}$ and $\mathrm{WO}_{70}$, the values of $\mathrm{R}^{2}$ were between $0.94-0.98, \mathrm{WO}_{50}$ and $\mathrm{WO}_{70}$ : volume of phosphate buffer solution required to wash out $50 \%$ and $70 \%$ of the formulations, respectively, NA: not applicable.

The reason for the better retention of thiolated nanoparticles compared to PEGylated and POZylated nanoparticles was most likely the formation of disulfide bridges between the thiolated nanoparticles and cysteine-rich domains of mucosal layers, since these nanoparticles had a greater free thiol content than PEGylated and POZylated nanoparticles. Conversely, 
PEGylated and POZylated nanoparticles were effectively removed after a few wash cycles probably due to less disulfide bridge formation by virtue of their lower thiol contents.

As all 3 types of the nanoparticles used in this study were negatively charged, the discrepancy in their mucoadhesive properties is unlikely to be related to the difference in charge. According to the electronic theory of mucoadhesion, anionic particles should not show any strong adhesion to mucosal surfaces, which are also negatively charged, as like charges usually repel each other. However, mucoadhesion can be the result of one or more mechanisms, including ionic interaction, covalent bonding, hydrogen bonding, hydrophobic interaction, wetting, adsorption, diffusion and entanglement (Smart, 2005). Additionally, as the particles are dispersed in water, the effect of dispersion media on the mucoadhesive properties of the particles was excluded. Finally, the mucoadhesion was studied at $\mathrm{pH} 6.8$, which was close to the $\mathrm{pH}$ of the ultrapure water used to dilute the nanoparticles for DLS size measurement. From the DLS data (Tables 1 and 2 and Fig. 1 and 4) it is clearly seen that the nanoparticles were stable at neutral $\mathrm{pH}$ and did not show any sign of aggregation. Therefore, their retention would not be due to their deposition on the mucosal surfaces and rather was due to their ability to form disulfide bonds with cysteine residues in mucus.

Several studies have shown that decorating nanoparticles with PEG facilitates their diffusion or penetration through mucus barriers (Lai et al., 2007; Mun et al., 2014b; Maisel et al., 2015). Lai et al. (2007) reported that PEGylation of conventional large polystyrene nanoparticles (200 and $500 \mathrm{~nm}$ ) led to their rapid transport through fresh human cervicovaginal mucus (CVM) and their diffusion coefficient $\left(D_{c}\right)$ in mucus was just 4 to 6 -fold smaller than in water. In contrast, the $\mathrm{D}_{\mathrm{c}}$ of uncoated polystyrene nanoparticles in CVM was 2,400 to 40,000-fold lower than in water. POZ has been studied as an alternative to PEG for the development of mucuspenetrating nanomaterials (Khutoryanskiy, 2018). For instance, Mansfield et al. (2015) showed that POZylated silica nanoparticles diffused rapidly through porcine gastric mucosa. Similarly, in this study, the PEGylated and POZylated nanoparticles showed less mucoadhesion than thiolated nanoparticles. Based on these and our previous findings, we postulate that the PEGylated and POZylated nanoparticles may diffuse through rat intestinal mucus and thus be mucus-penetrating, which is a desirable property in some drug delivery applications. In other words, the fact that the PEGylated and POZylated nanoparticles exhibited less mucosal retention may not diminish their importance as a drug delivery system relative to the more mucoadhesive thiolated nanoparticles. The selection of either mucoadhesive or mucuspenetrating carriers depends on the drug being delivered, the mucosal surface being targeted, 
the mucus turnover rate, the presence of water, the disease state and the intended release profile. Generally, mucoadhesive nanoparticles are advantageous for ocular and intravesical drug delivery where lacrimation in the eye and urine voiding from the bladder remove a substantial amount of the drug at the site of application/absorption. Mucus-penetrating carriers, on the other hand, are desirable in the design of oral drug delivery systems, where deeper mucus gel penetration and a broader particle distribution through the absorptive epithelia are required (Mun et al., 2014b; Maisel et al., 2015; Mun et al., 2016; Netsomboon \& Bernkop-Schnürch, 2016).

\section{Conclusion}

In this study, we have investigated the $\mathrm{pH}$-stability profiles of thiolated, PEGylated and POZylated silica nanoparticles and evaluated their mucoadhesive properties in rat intestinal mucosa. PEGylated nanoparticles showed similar size over a broad pH range (1.5-9). Also, in case of thiolated and POZylated nanoparticles, no change in size was observed at pH 2.5-9. Thiolated nanoparticles were mucoadhesive in rat intestinal mucosa ex vivo but to a lesser extent than to urinary bladder mucosa as reported in our previous study. These mucoadhesive nanoparticles can potentially be used to prolong drug release. PEGylated and POZylated nanoparticles showed less mucoadhesion, which suggests better permeability through the mucus layer compared to thiolated nanoparticles. This property may allow the particles to diffuse more easily through the mucus barrier to the deeper absorptive epithelia, leading to better absorption of the drugs.

\section{Acknowledgements}

We are thankful to HCED-Iraq for funding this research. We acknowledge the assistance of staff at the Chemical Analysis Facility (CAF), University of Reading, in FTIR, fluorescence spectroscopy and TEM experiments. We are grateful to Mr. Andrew Cripps for his assistance in obtaining animal tissues used in this study.

\section{References}


Bansil, R., \& Turner, B.S., 2006. Mucin structure, aggregation, physiological functions and biomedical applications. Curr. Opin. Colloid Interface Sci. 11, 164-170.

Bernkop-Schnürch, A., 2005. Mucoadhesive systems in oral drug delivery. Drug Discovery Today: Technol. 2, 8387.

Bonengel, S., \& Bernkop-Schnürch, A., 2014. Thiomers-from bench to market. J. Control. Release 195, 120-129.

Cook, M.T., Schmidt, S.A., Lee, E., Samprasit, W., Opanasopit, P., \& Khutoryanskiy, V.V., 2015. Synthesis of mucoadhesive thiol-bearing microgels from 2-(acetylthio)ethylacrylate and 2hydroxyethylmethacrylate: Novel drug delivery systems for chemotherapeutic agents to the bladder. J. Mater. Chem. B 3, 6599-6604.

Date, A.A., Hanes, J., \& Ensign, L.M., 2016. Nanoparticles for oral delivery: Design, evaluation and state-of-theart. J. Control. Release 240, 504-526.

Davoudi, Z., Peroutka-Bigus, N., Bellaire, B., Wannemuehler, M., Barrett, T.A., Narasimhan, B., \& Wang, Q., 2018. Intestinal organoids containing poly(lactic-co-glycolic acid) nanoparticles for the treatment of inflammatory bowel diseases. J Biomed Mater Res A 106, 876-886.

Deplancke, B., \& Gaskins, H.R., 2001. Microbial modulation of innate defense: Goblet cells and the intestinal mucus layer. Am. J. Clin. Nutr. 73, 1131S-1141S.

Dünnhaupt, S., Barthelmes, J., Hombach, J., Sakloetsakun, D., Arkhipova, V., \& Bernkop-Schnürch, A., 2011. Distribution of thiolated mucoadhesive nanoparticles on intestinal mucosa. Int. J. Pharm. 408, 191-199.

Dyab, A.K.F., 2012. Destabilisation of Pickering emulsions using pH. Colloids Surf. Physicochem. Eng. Aspects 402, 2-12.

Gao, L., Liu, G., Ma, J., Wang, X., Zhou, L., Li, X., \& Wang, F., 2013. Application of drug nanocrystal technologies on oral drug delivery of poorly soluble drugs. Pharm. Res. 30, 307-324.

Hu, R., Zheng, H., Cao, J., Davoudi, Z., \& Wang, Q., 2017. Synthesis and in vitro characterization of carboxymethyl chitosan-CBA-doxorubicin conjugate nanoparticles as $\mathrm{pH}$-sensitive drug delivery systems. Journal of Biomedical Nanotechnology 13, 1097-1105.

Irmukhametova, G.S., Fraser, B.J., Keddie, J.L., Mun, G.A., \& Khutoryanskiy, V.V., 2012. Hydrogen-bonding-driven self-assembly of PEGylated organosilica nanoparticles with poly(acrylic acid) in aqueous solutions and in layer-by-layer deposition at solid surfaces. Langmuir 28, 299-306.

Irmukhametova, G.S., Mun, G.A., \& Khutoryanskiy, V.V., 2011. Thiolated mucoadhesive and PEGylated nonmucoadhesive organosilica nanoparticles from 3-mercaptopropyltrimethoxysilane. Langmuir 27, 9551-9556.

Johansson, M.E.V., Ambort, D., Pelaseyed, T., Schütte, A., Gustafsson, J.K., Ermund, A., Subramani, D.B., HolménLarsson, J.M., Thomsson, K.A., Bergström, J.H., van der Post, S., Rodriguez-Piñeiro, A.M., Sjövall, H., Bäckström, M., \& Hansson, G.C., 2011. Composition and functional role of the mucus layers in the intestine. Cell. Mol. Life Sci. 68, 3635-3641.

Keely, S., Rullay, A., Wilson, C., Carmichael, A., Carrington, S., Corfield, A., Haddleton, D.M., \& Brayden, D.J., 2005. In vitro and ex vivo intestinal tissue models to measure mucoadhesion of poly (methacrylate) and $\mathrm{N}$-trimethylated chitosan polymers. Pharm. Res. 22, 38-49.

Kessler, T.L., \& Dartt, D.A. (1994). Neural stimulation of conjunctival goblet cell mucous secretion in rats, in: D. A. Sullivan (Ed.), Lacrimal gland, tear film, and dry eye syndromes: Basic science and clinical relevance. Springer US, Boston, MA, pp. 393-398.

Khutoryanskiy, V.V., 2011. Advances in mucoadhesion and mucoadhesive polymers. Macromol. Biosci. 11, 748764.

Khutoryanskiy, V.V., 2018. Beyond PEGylation: Alternative surface-modification of nanoparticles with mucusinert biomaterials. Adv. Drug Del. Rev. 124, 140-149.

Lai, S.K., O'Hanlon, D.E., Harrold, S., Man, S.T., Wang, Y.-Y., Cone, R., \& Hanes, J., 2007. Rapid transport of large polymeric nanoparticles in fresh undiluted human mucus. Proc. Natl. Acad. Sci. U. S. A. 104, 1482-1487.

Leal, J., Smyth, H.D.C., \& Ghosh, D., 2017. Physicochemical properties of mucus and their impact on transmucosal drug delivery. Int. J. Pharm. 532, 555-572.

Lehr, C.-M., Bouwstra, J.A., Kok, W., De Boer, A.G., Tukker, J.J., Verhoef, J.C., Breimer, D.D., \& Junginger, H.E., 1992. Effects of the mucoadhesive polymer polycarbophil on the intestinal absorption of a peptide drug in the rat. J. Pharm. Pharmacol. 44, 402-407.

Lozoya-Agullo, I., Zur, M., Beig, A., Fine, N., Cohen, Y., González-Álvarez, M., Merino-Sanjuán, M., GonzálezÁlvarez, I., Bermejo, M., \& Dahan, A., 2016. Segmental-dependent permeability throughout the small intestine following oral drug administration: Single-pass vs. Doluisio approach to in-situ rat perfusion. Int. J. Pharm. 515, 201-208. 
Lundquist, P., \& Artursson, P., 2016. Oral absorption of peptides and nanoparticles across the human intestine: Opportunities, limitations and studies in human tissues. Adv Drug Deliv Rev 106, 256-276.

Maisel, K., Ensign, L., Reddy, M., Cone, R., \& Hanes, J., 2015. Effect of surface chemistry on nanoparticle interaction with gastrointestinal mucus and distribution in the gastrointestinal tract following oral and rectal administration in the mouse. J. Control. Release 197, 48-57.

Malvern Instruments Ltd., 2011. Dynamic light scattering-common terms defined. https://www.malvern.com/en/support/resourcecenter/Whitepapers/WP111214DLSTermsDefined.ht $\mathrm{ml}$ (accessed 23 January 2018).

Mansfield, E.D. (2016). Synthesis, characterisation, and diffusive properties of functionalised nanomaterials. (PhD), University of Reading.

Mansfield, E.D., de la Rosa, V.R., Kowalczyk, R.M., Grillo, I., Hoogenboom, R., Sillence, K., Hole, P., Williams, A.C., \& Khutoryanskiy, V.V., 2016. Side chain variations radically alter the diffusion of poly(2-alkyl-2oxazoline) functionalised nanoparticles through a mucosal barrier. Biomaterials Science 4, 1318-1327.

Mansfield, E.D., Sillence, K., Hole, P., Williams, A.C., \& Khutoryanskiy, V.V., 2015. POZylation: A new approach to enhance nanoparticle diffusion through mucosal barriers. Nanoscale 7, 13671-13679.

Merchant, H.A., Rabbie, S.C., Varum, F.J., Afonso-Pereira, F., \& Basit, A.W., 2014. Influence of ageing on the gastrointestinal environment of the rat and its implications for drug delivery. Eur. J. Pharm. Sci. 62, 7685.

Moghissi, K., Neuhaus, O.W., \& Stevenson, C.S., 1960. Composition and properties of human cervical mucus. I. Electrophoretic separation and identification of proteins. The Journal of Clinical Investigation 39, 13581363.

Moustafine, R.I., Bobyleva V.L., Bukhovets A.V., Garipova V.R., Kabanova T.V., Kemenova V.A., Van den Mooter G., 2011. Structural transformations during swelling of polycomplex matrices based on countercharged (meth)acrylate copolymers (Eudragit ${ }^{\circledR}$ E PO/Eudragit ${ }^{\circledR}$ L 100-55). J. Pharm. Sci. 100, 874-885.

Mun, E.A., Hannell, C., Rogers, S.E., Hole, P., Williams, A.C., \& Khutoryanskiy, V.V., 2014a. On the role of specific interactions in the diffusion of nanoparticles in aqueous polymer solutions. Langmuir 30, 308-317.

Mun, E.A., Morrison, P.W., Williams, A.C., \& Khutoryanskiy, V.V., 2014b. On the barrier properties of the cornea: A microscopy study of the penetration of fluorescently labeled nanoparticles, polymers, and sodium fluorescein. Mol. Pharm. 11, 3556-3564.

Mun, E.A., Williams, A.C., \& Khutoryanskiy, V.V., 2016. Adhesion of thiolated silica nanoparticles to urinary bladder mucosa: Effects of PEGylation, thiol content and particle size. Int. J. Pharm. 512, 32-38.

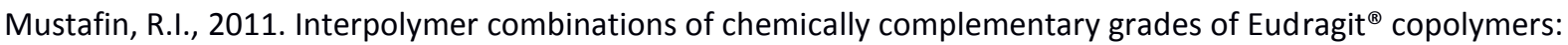
a new direction in the design of peroral solid dosage forms of drug delivery systems with controlled release (review), Pharm. Chem. J. 45, 285-295

Netsomboon, K., \& Bernkop-Schnürch, A., 2016. Mucoadhesive vs. mucopenetrating particulate drug delivery. Eur. J. Pharm. Biopharm. 98, 76-89.

Nguyen, T.X., Huang, L., Gauthier, M., Yang, G., \& Wang, Q., 2016. Recent advances in liposome surface modification for oral drug delivery. Nanomedicine (Lond). 11, 1169-1185.

Nielsen, L.S., Schubert, L., \& Hansen, J., 1998. Bioadhesive drug delivery systems I. Characterisation of mucoadhesive properties of systems based on glyceryl mono-oleate and glyceryl monolinoleate. Eur. J. Pharm. Sci. 6, 231-239.

Oltra-Noguera, D., Mangas-Sanjuan, V., González-Álvarez, I., Colon-Useche, S., González-Álvarez, M., \& Bermejo, M., 2015. Drug gastrointestinal absorption in rat: Strain and gender differences. Eur. J. Pharm. Sci. 78, 198-203.

Owens, D.E., \& Peppas, N.A., 2006. Opsonization, biodistribution and pharmacokinetics of polymeric nanoparticles. Int. J. Pharm. 307, 93-102.

Prego, C., Fabre, M., Torres, D., \& Alonso, M.J., 2006. Efficacy and mechanism of action of chitosan nanocapsules for oral peptide delivery. Pharm. Res. 23, 549-556.

Ranga Rao, K.V., \& Buri, P., 1989. A novel in situ method to test polymers and coated microparticles for bioadhesion. Int. J. Pharm. 52, 265-270.

Siew, A., Le, H., Thiovolet, M., Gellert, P., Schatzlein, A., \& Uchegbu, I., 2012. Enhanced oral absorption of hydrophobic and hydrophilic drugs using quaternary ammonium palmitoyl glycol chitosan nanoparticles. Mol. Pharm. 9, 14-28.

Smart, J.D., 2005. The basics and underlying mechanisms of mucoadhesion. Adv. Drug Del. Rev. 57, 1556-1568.

Sogias, I.A., Williams, A.C., \& Khutoryanskiy, V.V., 2008. Why is chitosan mucoadhesive? Biomacromolecules 9 , 1837-1842. 
Spicer, S.S., Schulte, B.A., \& Chakrin, L.W., 1983. Ultrastructural and histochemical observations of respiratory epithelium and gland. Exp. Lung Res. 4, 137-156.

Štorha, A., Mun, E.A., \& Khutoryanskiy, V.V., 2013. Synthesis of thiolated and acrylated nanoparticles using thiolene click chemistry: Towards novel mucoadhesive materials for drug delivery. RSC Adv. 3, 1227512279.

Symonds, B.L., Lindsay, C.I., Thomson, N.R., \& Khutoryanskiy, V.V., 2016a. Chitosan as a rainfastness adjuvant for agrochemicals. RSC Adv. 6, 102206-102213.

Symonds, B.L., Thomson, N.R., Lindsay, C.I., \& Khutoryanskiy, V.V., 2016b. Rainfastness of poly(vinyl alcohol) deposits on Vicia faba leaf surfaces: From laboratory-scale washing to simulated rain. ACS Appl. Mater. Interfaces 8, 14220-14230.

Tonglairoum, P., Brannigan, R.P., Opanasopit, P., \& Khutoryanskiy, V.V., 2016. Maleimide-bearing nanogels as novel mucoadhesive materials for drug delivery. J. Mater. Chem. B 4, 6581-6587.

Varum, F.J.O., Veiga, F., Sousa, J.S., \& Basit, A.W., 2010. An investigation into the role of mucus thickness on mucoadhesion in the gastrointestinal tract of pig. Eur. J. Pharm. Sci. 40, 335-341.

Varum, F.J.O., Veiga, F., Sousa, J.S., \& Basit, A.W., 2012. Mucus thickness in the gastrointestinal tract of laboratory animals. J. Pharm. Pharmacol. 64, 218-227.

Withers, C.A., Cook, M.T., Methven, L., Gosney, M.A., \& Khutoryanskiy, V.V., 2013. Investigation of milk proteins binding to the oral mucosa. Food Funct. 4, 1668-1674.

Yun, Y., Cho, Y.W., \& Park, K., 2013. Nanoparticles for oral delivery: Targeted nanoparticles with peptidic ligands for oral protein delivery. Adv. Drug Del. Rev. 65, 822-832. 